\title{
Embriopatía por isotretinoína. Una entidad que puede evitarse
} Isotretinoin embryopathy. An entity that can be avoided

\author{
Prof. Francisco Cammarata-Scalisi, ${ }^{a}$ Dra. Dairelis Nieves, ${ }^{b}$ Prof. Andrea Avendaño, ${ }^{a}$ \\ Prof. María A. Lacruz-Rengel, ${ }^{c}$ Br. Karelys Alviárez, ${ }^{d} B r$. Francys Dávila, ${ }^{d}$ Prof. Izzet Yavuze y \\ Dr. Michele Calleaf
}

\section{RESUMEN}

Laisotretinoína es el medicamento más efectivoen el tratamiento del acné noduloquístico recalcitrante grave. Sin embargo, el tratamiento con este fármaco se encuentra asociado con efectos adversos, y el más grave es la teratogénesis. Se ha estimado que $40 \%$ de los embarazos expuestos a isotretinoína presenta un aborto espontáneo y $35 \%$ desarrolla embriopatía. Se presenta el caso de un recién nacido con antecedente de exposición prenatal a isotretinoína, una entidad clínica que puede evitarse, con graves defectos congénitos en el sistema nervioso central eimportantes dismorfias faciales, con evolución clínica desfavorable.

Palabras clave: isotretinoína, teratogénesis, embriopatías.

\begin{abstract}
Isotretinoin is the most effective drug in the treatment of severe recalcitrant nodulocystic acne. However, treatment with this drug is associated with adverse effects, the most severe being teratogenesis. It has been estimated that $40 \%$ of pregnancies exposed to isotretinoin present spontaneous abortion and 35\% develop embryopathy. We present the case of a newborn with a history of prenatal exposure to isotretinoin, a clinical entity that can be avoided, with severe congenital defects in the central nervous system and important facial dysmorphisms, with unfavorable clinical course.
\end{abstract}

Key words: isotretinoin, teratogenesis, embryopathy.

http: / / dx.doi.org/10.5546/ aap.2018.e303

Cómo citar: Cammarata-Scalisi MA, Nieves D, Avendaño A, et al. Embriopatía por isotretinoína. Una entidad que puede evitarse. Arch Argent Pediatr 2018;116(2):e303-e307.

a. Unidad de Genética Médica, Departamento de Puericultura y Pediatría.

b. Residente del Posgrado de Puericultura y Pediatría.

c. Servicio de Neuropediatría, Departamento de Puericultura y Pediatría.

d. Estudiante de Medicina, Facultad de Medicina-

Universidad de Los Andes, Mérida, Venezuela.

e. Dicle University, Faculty of Dentistry, Department of Pediatric Dentistry, Diyarbakır, Turkey.

f. Unit of Dentistry, Bambino Gesù Children's Hospital, Institute for Research and Health Care (IRCCS), Rome, Italy.

Correspondencia:

Prof. Francisco Cammarata-Scalisi:

francocammarata19@gmail.com.

Financiamiento: Ninguno.

Conflicto de intereses: Ninguno que declarar.

Recibido: 12-6-2017

Aceptado: 12-10-2017

\section{INTRODUCCIÓN}

La isotretinoína (13-cis ácido retinoico) es una forma del análogo de la vitamina $\mathrm{A}, 1,2$ introducido en 1982 por Hoffmann-La Roche para el tratamiento del acné noduloquístico recalcitrante grave. ${ }^{1,3,4}$ Este fármaco interviene en la patogénesis del acné, como la actividad antiseborrea por la glándula sebácea, proliferación de queratinocitos, crecimiento del Propionibacterium acnes e inflamación. ${ }^{3}$ Sin embargo, su empleo está asociado con efectos adversos; el más grave es la teratogénesis, seguido de efectos mucocutáneos secundarios, la elevación de transaminasas e hipertrigliceridemia. ${ }^{1}$ Entre otros efectos adversos menos frecuentes, se pueden encontrar trastornos psiquiátricos, como la depresión y el riesgo de suicidio, además de la enfermedad inflamatoria intestinal. ${ }^{1,3,5,6}$

La isotretinoína incrementa el riesgo de abortos espontáneos y mortinatos en $40 \%$ de los embarazos con exposición al medicamento durante el primer trimestre y el riesgo de desarrollar embriopatía es de $35 \%$, si es expuesto más allá de la segunda semana de la concepción, ${ }^{4}$ y cuyos hallazgos clínicos se exponen en la Tabla 1.1,7,

Las principales características de la entidad se pueden explicar por el efecto del fármaco en un aumento en la apoptosis en las células de la cresta neural, al igual que el síndrome de alcohol fetal. ${ }^{1,9}$ La base molecular es aún desconocida, aunque la sobreexpresión del factor de transcripción p53 puede intervenir, como en el síndrome de Treacher-Collins, coloboma, cardiopatía, atresia de coanas, retraso psicomotor y del crecimiento, genitales anómalos, malformaciones auriculares $\mathrm{y} / \mathrm{o}$ sordera (coloboma, heart defects, atresia choanae, growth retardation, genital abnormalities, and ear abnormalities; CHARGE, por sus siglas en inglés) y el ya comentado síndrome de alcohol fetal, que exhiben dismorfias craneofaciales similares. ${ }^{9}$

Se presenta el caso de un recién nacido con antecedente de exposición prenatal a isotretinoína, una entidad clínica que puede evitarse, con graves defectos congénitos en el sistema nervioso central y dismorfias faciales, con evolución clínica desfavorable. 


\section{CASO CLÍNICO}

Recién nacido evaluado por presentar dismorfia facial y alteraciones en el sistema nervioso central por el estudio de ecografía transfontanelar. Correspondió a un caso único en la familia de la madre, y no se precisaron datos del padre.

Madre de 16 años de edad, primigesta, embarazo no planificado y mal controlado desde el quinto mes de gestación, complicado por un síndrome febril por dengue, con amenaza de parto pretérmino y diagnóstico prenatal de ventriculomegalia grave, agenesia de cuerpo calloso y polihidramnios por ecografía a las 27 semanas. Presentó como antecedente el uso de isotretinoína durante cuatro meses en el período periconcepcional. La serología para la sífilis (venereal disease research laboratory; VDRL, por sus siglas en inglés) fue no reactiva y el virus de la inmunodeficiencia humana (VIH) fue negativo. La inmunoglobulina $G$ ( $\operatorname{IgG}$ ) para rubeola fue positiva; sin embargo, la IgM fue negativa, así como la IgM e IgG para citomegalovirus y

\section{Toxoplasma gondii.}

Obtenido por cesárea segmentaria a las 28 semanas y seis días por ecografía, por inicio del parto pretérmino. La presentación fue cefálica, requirió reanimación, que ameritó la hospitalización en la Unidad de Alto Riesgo Neonatal, con test de Apgar de 5 y 6 puntos al primer y quinto minuto, respectivamente. El peso al nacer fue de $1540 \mathrm{~g}$ (puntaje de desvío estándar $-\mathrm{SDE}-4,4)$; la talla al nacer, de $41 \mathrm{~cm}$ (SDE -7,3); y el perímetro cefálico, de $29 \mathrm{~cm}(\mathrm{P}<3)$. La edad gestacional por examen físico (test de Ballard) fue de 29 semanas.

Presentó cianosis con posterior diagnóstico de síndrome de dificultad respiratoria por enfermedad de la membrana hialina, el test de Silverman-Anderson con 5 puntos (tiraje intercostal: 2 puntos; retracción esternal: 2 puntos; y quejido espiratorio: 1 punto), y requirió ventilación mecánica durante 40 horas, en modo de ventilación intermitente obligatoria sincronizada con parámetros ventilatorios mínimos, con adecuada tolerancia posterior a

TABLA 1. Hallazgos clinicos en la embriopatía por isotretinoína ${ }^{1,7,8}$

\begin{tabular}{llc}
\hline Sistema u órgano & Hallazgo & Paciente \\
\hline Nervioso & Microcefalia & + \\
& Hidrocefalia & + \\
& Holoprosencefalia & - \\
& Hipoplasia cerebelar & - \\
& Agenesia de vermis cerebeloso & - \\
& Trastornos de migración neuronal & NP \\
Anomalías del tracto corticoespinal & - \\
Dismorfias faciales & Defecto de cierre del tubo neural & NP \\
& Retardo mental & - \\
& Parálisis facial & + \\
Aimo & Hipertelorismo ocular & + \\
\hline & Puente nasal deprimido & + \\
Paladar ojival & + \\
Cardiovascular & Micrognatia & - \\
& Anotia & + \\
& Microtia & - \\
& Atresia del canal auditivo externo & + \\
& Estenosis del canal auditivo externo & - \\
& Colesteatoma & NP
\end{tabular}

NP: no precisado. 
la extubación de forma programada, adecuado patrón ventilatorio y equilibrio ácido-base corroborado por gasometría arterial. Permaneció recibiendo oxígeno húmedo por cámara cefálica con el sistema Blender. Recibió ampicilina y gentamicina por la invasión de la ventilación mecánica, sin ameritar la rotación de antibióticos.

Al momento del examen físico de ingreso, se encontraron, como hallazgos positivos, microcefalia, fontanela anterior amplia de $4 \times 3 \mathrm{~cm}$ y posterior de $2 \times 1 \mathrm{~cm}$, prominencia frontal, leve hipertricosis, hipertelorismo ocular, puente nasal amplio y cóncavo, micrognatia, pabellones auriculares de implantación baja, displásicos y microtia bilateral (Figura 1). La piel se mostraba atrófica, permitía visualizar los elementos vasculares y era redundante en los miembros inferiores.

La ecografía transfontanelar evidenció hidrocefalia y agenesia de cuerpo calloso, el cual fue confirmado por la tomografía computarizada cerebral (Figura 2). La evaluación cardiovascular fue normal y la ecocardiografía transtorácica no encontró alteración estructural. La ecografía renal indicó pielectasia derecha. Fue evaluado por el Servicio de Oftalmología Pediátrica, que refirió un fondo de ojo sin alteraciones, y de Otorrinolaringología por microtia grado II.

El paciente presentó hipotermia de origen central. El sexto día, tuvo episodios de apnea en múltiples oportunidades, para lo cual se instauró una terapia con aminofilina y se conectó a ventilación mecánica no invasiva tipo presión positiva continua por vía nasal, con discreta mejoría, pero, el día siguiente, presentó un episodio de apnea de origen central, con paro cardiorrespiratorio, que no respondió a las maniobras de reanimación básica y avanzada.

FIGURA 2. La tomografía computarizada cerebral evidenció hidrocefalia

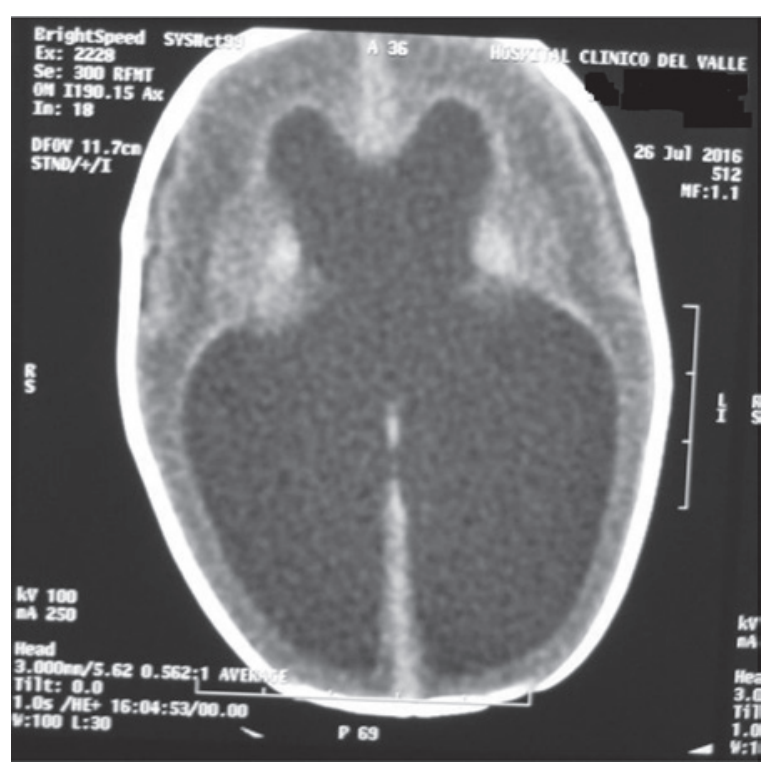

FIGURA 1. Prominencia frontral, leve hipertricosis, puente nasal cóncavo, micrognatia, pabellones auriculares de implantación baja, displásicos y microtia bilateral
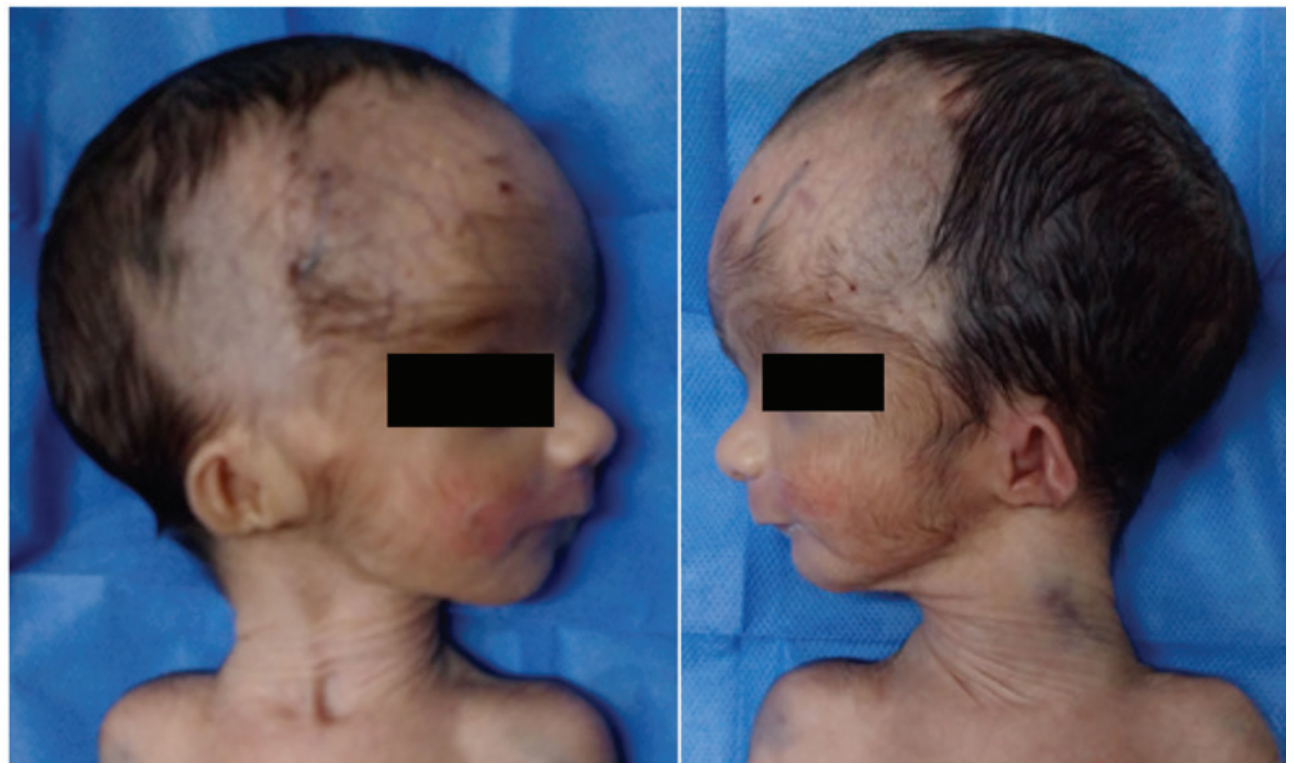


\section{DISCUSIÓN}

Un teratógeno es todo agente externo que llega al embrión o feto en desarrollo y es capaz de causar, en forma directa o indirecta, defectos congénitos o alteraciones funcionales (bioquímicas, metabólicas, hormonales, inmunológicas, crecimiento y comportamiento), que, incluso, pueden afectar después del nacimiento. ${ }^{3}$ La isotretinoína, como ya se hizo referencia, es un teratógeno humano cuya exposición en el útero puede ocasionar múltiples malformaciones congénitas. La constelación de hallazgos clínicos es conocida como embriopatía por isotretinoína. ${ }^{2}$ La teratogenicidad de este fármaco fue clasificada como categoría X en el embarazo antes de su lanzamiento. ${ }^{3}$ La exposición entre la segunda y la quinta semana después de la concepción es considerada el período crítico. Sin embargo, en cualquier momento del embarazo, presenta alto riesgo de teratogenicidad. ${ }^{2}$

La muerte celular programada (apoptosis) contribuye evolutivamente en el desarrollo del sistema nervioso y requiere de un equilibrio regulado en la señalización durante gran parte de los períodos críticos, lo cual asegura una diferenciación y maduración celular apropiada. El ácido transretinoico conduce a la reprogramación a gran escala de la expresión de los genes de las células de la cresta neural cerebral, que lleva a un aumento de la apoptosis. La muerte celular excesiva puede afectar a los neuroblastos del ganglio del trigémino de origen de la placoda $\mathrm{y}$, de continuar con la administración de la isotretinoína en el momento de la formación del ganglio, conduce a malformaciones. ${ }^{1}$

Además, la morfogénesis y la remodelación del desarrollo de los tejidos cardiovasculares implican la regulación coordinada de la proliferación celular y la apoptosis. ${ }^{1}$ Existe una clara evidencia de que la apoptosis focal contribuye al desarrollo temprano de la vía de salida embrionaria, válvulas cardíacas, sistema de conducción y el desarrollo de la vasculatura coronaria. ${ }^{3}$ Aunque, en este caso, no se encontraron alteraciones cardiovasculares, se desconocen las causas de la expresividad variable en el fenotipo de los afectados.

También, este fármaco tiene un efecto antineuroblastoma y juega un papel en el tratamiento de alto riesgo de neuroblastoma. Hasta el momento, no se ha asociado la embriopatía por isotretinoína con el incremento en la prevalencia de neoplasias. Sin embargo, un caso de neuroblastoma congénito metastásico asociado con esta embriopatía ha sido documentado. $^{2}$

Es por ello por lo que se recomienda a las mujeres en edad fértil usar anticonceptivos orales un mes antes de iniciar la isotretinoína y evitar quedar embarazadas un mes después de completar el tratamiento. Las advertencias médicas deben ser cada vez más detalladas y se debe firmar un consentimiento informado para el uso del medicamento. En 2006, la Administración de Medicamentos y Alimentos, mediante la implementación del programa iPLEDGE, aumentó la vigilancia, en la cual se encontraba el asesoramiento mensual de riesgo con realización de pruebas de embarazo. ${ }^{3,6}$ Por su parte, en Argentina, la Administración Nacional de Medicamentos, Alimentos y Tecnología Médica (ANMAT) lo incluye en los que están sujetos a farmacovigilancia intensiva (Disposición $\mathrm{N}^{\mathrm{o}} 2552$ /95), con necesidad de utilizar, al menos, dos métodos anticonceptivos e, igualmente, se debe firmar el consentimiento informado (Disposición ANMAT No 6083/09).

Además de estas recomendaciones de vigilancia, otras medidas educacionales dirigidas a la población en edad fértil deben ser dirigidas con el objeto de evitar los casos de embriopatía por isotretinoína. Se debe, igualmente, realizar un adecuado seguimiento en los casos de riesgo y brindar las opciones terapéuticas disponibles, lo que garantiza una evaluación médica interdisciplinaria.

Se presentó un caso clínico de embriopatía por isotretinoína, entidad clínica que podía evitarse, con malformaciones congénitas graves en el sistema nervioso central y dismorfias craneofaciales, que tuvo una evolución clínica desfavorable con fallecimiento en el período neonatal.

\section{Agradecimientos}

A la Licenciada Rosalía Gumina F., directora de la Biblioteca del Instituto Autónomo Hospital Universitario de Los Andes, Universidad de Los Andes.

\section{REFERENCIAS}

1. Melnik BC. Apoptosis may explain the pharmacological mode of action and adverse effects of isotretinoin, including teratogenicity. Acta Derm Venereol 2017;97(2):173-81.

2. AguilarS, Louis C, Hicks J, et al. Congenital neuroblastoma in a neonate with isotretinoinembryopathy.JPediatr Hematol Oncol 2014;36(2):e75-7.

3. Bauer LB, Ornelas JN, Elston DM, et al. Isotretinoin: controversies, facts, and recommendations. Expert Rev Clin Pharmacol 2016;9(11):1435-42. 
4. Pachajoa H, Ordoñez A. Embriopatía por isotretinoína con microtia-anotia y cardiopatía. Presentación de un caso. Arch Argent Pediatr 2012;110(3):e47-9.

5. Vallerand IA, Lewinson RT, Farris MS, et al. Efficacy and adverse events of oral isotretinoin for acne: a systematic review. Br J Dermatol 2017. [En prensa].

6. Karadag AS, Parish LC, Lambert WC. Isotretinoin in retrospect. Clin Dermatol 2017;35(3):335-9.
7. Troncoso Sch M, Rojas HC, Bravo CE. Embriopatía por isotretinoína: un daño evitable. Rev Med Chile 2008;136(6):763-6.

8. Van Abel KM, Nelson ME, Collar RM, et al. Development of canal cholesteatoma in a patient with prenatal isotretinoin exposure. Int J Pediatr Otorhinolaryngol 2010;74(9):1082-4.

9. Melnik BC. Over-expression of p53 explains isotretinoin's teratogenicity. Exp Dermatol 2017. [En prensa]. 